\title{
The Asymptotic Entanglement Cost of Preparing a Quantum State
}

\author{
Patrick M. Hayden ${ }^{1}$, Michał Horodecki ${ }^{2}$ and Barbara M. Terhal ${ }^{3}$ \\ ${ }^{1}$ Centre for Quantum Computation, Clarendon Laboratory, Parks Road, Oxford, OX1 3PU, UK; \\ ${ }^{2}$ Institute of Theoretical Physics and Astrophysics, University of Gdańsk, 80-952, Gdańsk, Poland; \\ ${ }^{3}$ IBM Watson Research Center, P.O. Box 218, Yorktown Heights, NY 10598, US. \\ Emails: patrick.hayden@qubit.org, michalh@iftia.univ.gda.pl, terhal@watson.ibm.com
}

(October 27, 2018)

\begin{abstract}
We give a detailed proof of the conjecture that the asymptotic entanglement cost of preparing a state $\rho$ is equal to $\lim _{n \rightarrow \infty} E_{f}\left(\rho^{\otimes n}\right) / n$ where $E_{f}$ is the entanglement of formation.
\end{abstract}

\section{INTRODUCTION}

One of the central issues in quantum entanglement theory is to determine how to optimally convert between different entangled states shared by distant observers Alice and Bob. More precisely, one is interested in the conversion of $m$ pairs of particles, with each pair in a state $\rho$, into $n$ pairs, each in another state $\rho^{\prime}$, by means of local quantum operations and classical communication (LOCC) [1] , so that the asymptotic ratio $m / n$ is minimal. Of course, the perfect transformation by LOCC

$$
\rho^{\otimes m} \rightarrow \rho^{\prime \otimes n}
$$

is usually impossible. Therefore, one permits imperfections and requires only asymptotically perfect transformations: the state $\rho^{\otimes m}$ is transformed into some state $\rho_{n}^{\prime}$, which for large $n$ approaches $\rho^{\prime \otimes n}$ with a chosen distance measure $D$ :

$$
\lim _{n \rightarrow \infty} D\left(\rho_{n}^{\prime}, \rho^{\prime \otimes n}\right)=0 .
$$

If the final state $\rho$ is a two-qubit singlet state $\frac{1}{\sqrt{2}}(|01\rangle-|10\rangle)$ (which we will denote by $\left.\left|\Psi_{-}\right\rangle\right)$, then the process of conversion is called distillation [2]. If, instead, it is the initial state that is in the form of singlets, then one refers to formation [1]. In this paper we are interested in the latter process. We will call the optimal asymptotic yield the entanglement cost, and denote it by $E_{c}$. In Ref. [3] it was shown that for a pure state $\rho=|\psi\rangle\langle\psi|, E_{c}$ is equal to the entropy of either of its reductions, e.g. $\rho_{A}=\operatorname{Tr}_{B}(\rho)$. Thus, to produce $|\psi\rangle^{\otimes n}$ one needs $m \approx n S\left(\rho_{A}\right)$ singlets, i.e. the initial state has to be $\left|\Psi_{-}\right\rangle^{\otimes n S\left(\rho_{A}\right)}$.

That result suggested the following stochastic method for the production of $\rho$ out of singlets [1]. One decomposes $\rho$ into an ensemble $\rho=\sum_{i} p_{i}\left|\psi_{i}\right\rangle\left\langle\psi_{i}\right|$ of pure states. Then one picks a state $\left|\psi_{i}\right\rangle$ according to the probability distribution $\left\{p_{i}\right\}$, makes the state $\left|\psi_{i}\right\rangle$ from initially shared singlets, and finally forgets the identity of the state. Therefore, one needs on average $\sum_{i} p_{i} S\left(\rho_{i}^{A}\right)$ singlets (where $\rho_{i}^{A}$ is the reduction of $\left|\psi_{i}\right\rangle$ ). One can choose the ensemble which minimizes the above average, with the corresponding minimal cost being called the entanglement of formation of $\rho$ [1], which we will denote by $E_{f}(\rho)$.

The above scenario can be improved if we realize that it might be more economical to produce the state $\rho^{\otimes n}$ all at once than it is to produce its $n$ constituents one by one (see e.g. [4]). Thus, the proposed optimal cost should be revised to 5

$$
E_{f}^{\infty}(\rho)=\lim _{n \rightarrow \infty} \frac{E_{f}\left(\rho^{\otimes n}\right)}{n} .
$$

(In the following we will argue that the limit on the right-hand-side of this equation exists.) This quantity is believed to be equal to the entanglement cost of preparing the state $\rho, E_{c}(\rho)$. The definition of the entanglement cost, however, refers to a deterministic number of input singlets, while in the stochastic method the number of input singlets is a random variable. Furthermore it is not clear that the stochastic method is really the optimal way to produce the state $\rho$. In this paper we resolve these issues by proving that $E_{f}^{\infty}(\rho)$ is equal to the entanglement cost $E_{c}(\rho)$. Our result is in a sense dual to that of Rains [6] concerning the entanglement of distillation. He showed that the entanglement of distillation, if defined in a way analogous to our definition of the entanglement cost, is equal to the expected yield of a stochastic protocol for generating output singlets. 
To begin, let us sketch our approach. In our proof, we will first show that $E_{f}^{\infty}(\rho) \geq E_{c}(\rho)$, i.e. that there exists a formation protocol that achieves the asymptotic rate $\frac{m}{n} \approx E_{f}^{\infty}(\rho)$, by explicitly constructing such a protocol based on the law of large numbers in both its classical and quantum [7 forms. Next, we will show that $E_{f}^{\infty}(\rho) \leq E_{c}(\rho)$. The latter inequality will be derived using only some general properties of the entanglement of formation, such as its monotonicity under LOCC operations and its quite strong continuity properties, proved by Nielsen [8]. Such an approach, focussing on abstract features of quantum information-theoretic quantities, has already proven to be fruitful in the domain of quantifying entanglement as well as in the study of quantum channel capacities [9, 10], providing a simple view of intrincate topics. To illustrate its power we will now sketch the proof of the inequality $E_{f}^{\infty}(\rho) \leq E_{c}(\rho)$.

As mentioned before, the entanglement of formation is monotonically decreasing under LOCC operations [1], i.e.

$$
E_{f}(\rho) \geq E_{f}(\mathcal{L}(\rho))
$$

for any state $\rho$ and LOCC operation $\mathcal{L}$. Moreover, the entanglement of formation is continuous $[8]$ in the sense that for states $\rho$ and $\rho^{\prime}$ we have

$$
\left|E_{f}(\rho)-E_{f}\left(\rho^{\prime}\right)\right| \leq 5 D\left(\rho, \rho^{\prime}\right) \log _{2} \operatorname{dim} \mathcal{H}+2 \eta\left(D\left(\rho, \rho^{\prime}\right)\right),
$$

where $\rho$ and $\rho^{\prime}$ are supported on the Hilbert space $\mathcal{H}$ and $\eta(x)=-x \log _{2} x$, under the assumption that $D\left(\rho, \rho^{\prime}\right)$ is sufficiently small. $D$ is the Bures distance, given by $D\left(\rho, \rho^{\prime}\right)=2 \sqrt{1-F\left(\rho, \rho^{\prime}\right)}$ with $F\left(\rho, \rho^{\prime}\right)=\operatorname{Tr} \sqrt{\rho^{1 / 2} \rho^{\prime} \rho^{1 / 2}}$ (Note that the normalization factor for $D$ is not completely standardized. We have made our choice to agree with Ref. [8]). The function $F$ is called the Uhlmann fidelity or square-root-fidelity [11, 12. Inequality (3) implies that if two states are close to one another, then so are their densities of entanglement. A similar continuity result has been proved in Ref. [13] for the relative entropy of entanglement [14].

Now, in order to prove $E_{f}^{\infty} \leq E_{c}$, consider the optimal formation protocol, i.e. the sequence of LOCC operations $\Lambda_{n}$ producing states $\rho_{n} \approx \rho^{\otimes n}$ out of $\left|\Psi_{-}\right\rangle^{\otimes m}$, so that $m / n$ tends to $E_{c}$. If we take large $n$, so that $D\left(\rho^{\otimes n}, \rho_{n}\right)$ is small, then by continuity (3) we have

$$
\frac{E_{f}\left(\rho^{\otimes n}\right)}{n} \approx \frac{E_{f}\left(\rho_{n}\right)}{n}
$$

Since $\rho_{n}=\Lambda_{n}\left(\left|\Psi_{-}\right\rangle^{\otimes m}\right)$, then from the monotonicity of $E_{f}$ we obtain

$$
\frac{E_{f}\left(\rho_{n}\right)}{n} \leq \frac{E_{f}\left(\left|\Psi_{-}\right\rangle\left\langle\left.\Psi_{-}\right|^{\otimes m}\right)\right.}{n}=\frac{m}{n}
$$

Now, by defnition, the left-hand-side of the equation (4) tends to $E_{f}^{\infty}$ while the last term of estimate (5) tends to $E_{c}$, hence we obtained the required inequality.

Since we used only two properties of the entanglement of formation, we can rephrase the result in a more general setting. Consider any function $f$, which can be regularized, i.e. for which the limit $f^{\infty}(\rho)=\lim _{n \rightarrow \infty} \frac{f\left(\rho^{\otimes n}\right)}{n}$ exists. Now, if $f$ is monotonic and continuous in the sense of equation (3), then $f^{\infty}$ is a lower bound for $E_{c}$. This supports the view, according to which the dual measures of entanglement, entanglement of distillation $E_{D}$ and entanglement cost $E_{c}$ are in a sense extreme ones [9].

\section{ENTANGLEMENT COST AND ENTANGLEMENT OF FORMATION}

Let us now pass to the fully rigorous part of the paper. Analogous to the definition of asymptotic reducibility in Ref. [15] (cf. [16]), we define the asymptotic entanglement cost for the preparation of a bipartite state to be

$$
E_{c}(\rho)=\inf \left\{E|\forall \epsilon>0, \delta>0, \exists m, n, \mathcal{L},| E-\frac{m}{n} \mid \leq \delta \text { and } D\left(\mathcal{L}\left(\left|\Psi_{-}\right\rangle\left\langle\left.\Psi_{-}\right|^{\otimes m}\right), \rho^{\otimes n}\right) \leq \epsilon\right\},\right.
$$

where $\left|\Psi_{-}\right\rangle$is the singlet state in $\mathbf{C}_{2} \otimes \mathbf{C}_{2}, \mathcal{L}$ is an LOCC superoperator and $D$ is again the Bures distance.

Our main result is the following:

Theorem 1 The asymptotic entanglement cost of preparing a state $\rho$ is given by

$$
E_{c}(\rho)=\lim _{n \rightarrow \infty} \frac{E_{f}\left(\rho^{\otimes n}\right)}{n}
$$


where $E_{c}(\rho)$ is defined in Eq. (6) and $E_{f}(\rho)$ is the entanglement of formation of $\rho$, defined as

$$
E_{f}(\rho)=\min _{\mathcal{E}=\left\{p_{i},\left|\psi_{i}\right\rangle\right\}} \sum_{i} p_{i} E\left(\left|\psi_{i}\right\rangle\left\langle\psi_{i}\right|\right)
$$

and $E(|\psi\rangle\langle\psi|)$ is the von Neumann entropy of the reduced density matrix of $|\psi\rangle$.

To make sense of the above claim, we note that the sequence $\left(\frac{E_{f}\left(\rho^{\otimes n}\right)}{n}\right)$ has a well-defined limit. This is a consequence of the fact that if a sequence $\left(a_{n}\right)$ satisfies $a_{n} \leq c n$ for some constant $c$ and $a_{n}+a_{m} \geq a_{n+m}$ for any $m, n$, then $\left(a_{n} / n\right)$ is convergent 17]. It is easy to see that our sequence satisfies the conditions.

We begin by proving that $E_{c}$ obeys a form of additivity that is highly desirable for an asymptotic cost function, and that will be useful in what follows.

Lemma $1 E_{c}\left(\rho^{\otimes k}\right)=k E_{c}(\rho)$ for all $k=1,2, \ldots$

Proof One direction is simple: since a protocol to approximate $\rho^{\otimes k}$ is just a protocol to approximate $k$ copies of $\rho$, $E_{c}\left(\rho^{\otimes k}\right) \geq k E_{c}(\rho)$. The first step in demonstrating that the inequality holds in the opposite direction will be to show that in the definition of $E_{c}, n$ can be taken to be arbitrarily large. Suppose not, in other words, that for fixed $\epsilon, \delta>0$, it is impossible to choose $n \geq N, m$, and $\mathcal{L}$ such that $\left|E_{c}(\rho)-m / n\right| \leq \delta$ and $D\left(\mathcal{L}\left(\left|\Psi_{-}\right\rangle\left\langle\Psi_{-}\right|{ }^{\otimes m}\right), \rho^{\otimes n}\right) \leq \epsilon$. By the definition of $E_{c}(\rho)$ and the fact that the set of LOCC operations is closed, this implies, however, that there exist $n<N, m$, and $\mathcal{L}$ such that $E_{c}(\rho)=m / n$ and $\mathcal{L}\left(\left|\Psi_{-}\right\rangle\left\langle\left.\Psi_{-}\right|^{\otimes m}\right)=\rho^{\otimes n}\right.$. Setting $r=\lceil N / n\rceil$ and applying $\mathcal{L}^{\otimes r}$ to $\left|\Psi_{-}\right\rangle\left\langle\left.\Psi_{-}\right|^{\otimes m r}\right.$ then violates the assumption that $n$ could not be taken arbitrarily large.

Now, to complete the proof, we will pick $n \gg k$ in order to efficiently approximate some number of copies of $\rho^{\otimes k}$. Formally, we write $n=r k+s$, where $r$ and $s$ are non-negative integers and $s<k$. If we produce an approximation to within $\epsilon$ of $\rho^{\otimes n}$ starting from $m$ singlets and then throw away the extra $s$ copies of $\rho$, we are left with an approximation to within $\epsilon$ of $\rho^{\otimes r k}$ that still required $m$ singlets. The waste, however, becomes insignicant since $|E-m / n| \rightarrow|E-m / r k|$ as $n \rightarrow \infty$.

Note that full additivity of $E_{c}(\rho)$, i.e. $E_{c}(\rho \otimes \sigma)=E_{c}(\rho)+E_{c}(\sigma)$ would be a stronger statement, possibly requiring additivity of $E_{f}$. With that technical lemma concluded, Theorem 1 becomes a consequence of the following two lemmas.

Lemma 2 Let the entanglement of formation of a (finite dimensional) density matrix $\rho$ be $E_{f}(\rho)=\sum_{i} p_{i} E_{f}\left(\left|\psi_{i}\right\rangle\left\langle\psi_{i}\right|\right)$ where the optimal ensemble is given by $\left\{p_{i},\left|\psi_{i}\right\rangle\right\}_{i=1}^{k}$. We have $E_{f}(\rho) \geq E_{c}(\rho)$.

Proof In the limit of large $n$, we will approximate $\rho^{\otimes n}$ by

$$
\rho_{T_{\delta_{1}}^{(n)}}=\sum_{s \in T_{\delta_{1}}^{(n)}}\left|\psi_{s}\right\rangle\left\langle\psi_{s}\right|
$$

where $T_{\delta_{1}}^{(n)}$ is the (strongly) typical set defined as follows. All states $\left|\psi_{s}\right\rangle$ (unnormalized) in this set are tensor products of states $\sqrt{p_{i}}\left|\psi_{i}\right\rangle$ and they are such that every state $\sqrt{p_{i}}\left|\psi_{i}\right\rangle$ occurs $p_{i} n \pm \delta_{1} n \log _{p_{i}}(2) / k$ times in the tensor product. (We assume that $p_{i}<1$ for all $i$. Otherwise, $\rho$ is a pure state.) This implies that the total probability $p_{s}=\left\langle\psi_{s} \mid \psi_{s}\right\rangle$ for a state $\left|\psi_{s}\right\rangle$ is bounded as

$$
2^{\left.-n(H(\vec{p}))+\delta_{1}\right)} \leq p_{s} \leq 2^{\left.-n(H(\vec{p}))-\delta_{1}\right)},
$$

where $H(\vec{p})$ is the Shannon entropy of $\vec{p}=\left(p_{i}\right)$. The density matrix $\rho_{T_{\delta}^{(n)}}$ is basically the state $\rho^{\otimes n}$ constructed by cutting off the unlikely sequences of states and then renormalizing [18]. In order to bound the fidelity for this approximation, we add these unlikely sequences of states with probability 0 in the sum. For any $\delta_{1}>0, \epsilon_{1}>0$ and $n$ sufficiently large we have by the asymptotic equipartition theorem [19] that the total probability $p_{T_{\delta_{1}}^{(n)}}$ for the typical set is larger than $1-\epsilon_{1}$, where $\epsilon_{1} \rightarrow 0$ as $n \rightarrow \infty$. Thus, using the joint concavity of $F$ [11], we can bound

$$
F\left(\rho^{\otimes n}, \rho_{T_{\delta_{1}}^{(n)}}\right) \geq \sqrt{1-\epsilon_{1}} .
$$

Consider the density matrix $\rho_{T_{\delta_{1}}^{(n)}}$ and its decomposition in terms of the states $\left|\psi_{s}\right\rangle$. Each state $\left|\psi_{s}\right\rangle$ can be obtained from a set of EPR pairs by entanglement dilution [3]. In particular, let $\left|\psi_{s}\right\rangle$ be a state in which every state $\left|\psi_{i}\right\rangle$ occurs $p_{i} n \pm \delta_{1} n \log _{p_{i}}(2) / k$ times. Starting from a set of maximally entangled EPR pairs, we do entanglement dilution 
for each state $\left|\psi_{i}\right\rangle^{\otimes p_{i} n \pm \delta_{1} n \log _{p_{i}}(2) / k}$. For any $\delta_{2}$ and $\epsilon_{2}$ greater than zero there exists an $n$ such that starting from $\left(p_{i} n+\delta_{1} n \log _{p_{i}}(2) / k\right)\left[E_{f}\left(\left|\psi_{i}\right\rangle\left\langle\psi_{i}\right|\right)+\delta_{2}\right]$ EPR pairs we can obtain an approximation to $\left|\psi_{i}\right\rangle^{\otimes p_{i} n \pm \delta_{1} n \log _{p_{i}}(2) / k}$ which has square-root-fidelity larger than $1-\epsilon_{2}$. Since there are $k$ states in the optimal ensemble (and $k$ is finite), we can therefore approximate the state $\left|\psi_{s}\right\rangle$ with square-root-fidelity $\left|\left\langle\psi \mid \psi^{\prime}\right\rangle\right|>\left(1-\epsilon_{2}\right)^{k}$, starting from $n \sum_{i} p_{i} E_{f}\left(\left|\psi_{i}\right\rangle\left\langle\psi_{i}\right|\right)+$ $n\left(O\left(\delta_{1}\right)+O\left(\delta_{2}\right)\right)$ EPR pairs, with $\epsilon_{2} \rightarrow 0, \delta_{1} \rightarrow 0$, and $\delta_{2} \rightarrow 0$ for $n \rightarrow \infty$.

The approximation of $\rho_{T_{\delta_{1}}^{(n)}}$ by $\rho_{T_{\delta_{1}}^{(n)}}^{\prime(n)}=\sum_{s \in T_{\delta_{1}}^{(n)}}\left|\psi_{s}^{\prime}\right\rangle\left\langle\psi_{s}^{\prime}\right|$, where $\left|\psi_{s}^{\prime}\right\rangle$ is the approximation of $\left|\psi_{s}\right\rangle$ which we obtain by entanglement dilution starting from the set of EPR pairs, has the property that

$$
F\left(\rho_{T_{\delta_{1}}^{(n)}}, \rho_{T_{\delta_{1}}^{(n)}}^{\prime}\right) \geq \sum_{s \in T_{\delta_{1}}^{(n)}}\left|\left\langle\psi_{s} \mid \psi_{s}^{\prime}\right\rangle\right| \geq\left(1-\epsilon_{2}\right)^{k} \equiv 1-\epsilon_{3},
$$

where $\epsilon_{3} \rightarrow 0$ for $n \rightarrow \infty$, since $k$ is finite. Furthermore, since we can make every state $\left|\psi_{s}^{\prime}\right\rangle$ starting from a given set of EPR pairs, we can make any convex combination, for example $\rho_{T_{\delta_{1}}^{(n)}}^{\prime}$, of the states $\left|\psi_{s}^{\prime}\right\rangle$ (see [20] and also [21]), starting from this same set of EPR pairs.

Finally, we can use the triangle inequality for the Bures metric, and Eqs. (11) and (12) to obtain that

$$
D\left(\rho^{\otimes n}, \rho_{T_{\delta_{1}}^{(n)}}^{\prime}\right) \leq 2 \sqrt{1-\sqrt{1-\epsilon_{1}}}+2 \sqrt{\epsilon_{3}} .
$$

which is the desired result.

The lemma can be applied to bound $E_{c}(\rho)$ from above by $E_{f}\left(\rho^{\otimes k}\right) / k$ where $k$ is any fixed number of copies of $\rho$, using Lemma 1. Consequently, we have that

$$
E_{f}^{\infty}(\rho) \geq E_{c}(\rho)
$$

Let us now prove the converse of this relation.

Lemma $3 E_{f}^{\infty}(\rho) \leq E_{c}(\rho)$.

Proof The basic idea of the proof, as sketched in the introduction, is to use the continuity of the entanglement of formation [8] and its monotonicity under LOCC operations. Throughout the proof we will use the notation $A_{n}=\frac{E_{f}\left(\rho^{\otimes n}\right)}{n}$. Now suppose that the lemma does not hold, so that $\lim A_{n}>E_{c}(\rho)$. It follows that there exists an integer $N$, such that for all $k>N$,

$$
\left|A_{k}-\lim A_{n}\right|<\Delta=\frac{\lim A_{n}-E_{c}(\rho)}{4}>0 .
$$

Let $\rho_{k}=\mathcal{L}\left(\left|\Psi_{-}\right\rangle\left\langle\left.\Psi_{-}\right|^{\otimes m}\right)\right.$ be an approximation to $\rho^{\otimes k}$. Now, supposing that $\rho$ is supported on Hilbert space $\mathcal{H}$, fix $\epsilon$ such that $5 \epsilon \log \operatorname{dim} \mathcal{H}-2 \epsilon \log \epsilon=\Delta$. It then follows from Eqs. (3) and our choice of $\epsilon$ that if $D\left(\rho^{\otimes k}, \rho_{k}\right)<\epsilon$ then

$$
\left|E_{f}\left(\rho^{\otimes k}\right)-E_{f}\left(\rho_{k}\right)\right|<k \Delta .
$$

Now we will apply the definition of $E_{c}(\rho)$ to fix $k$. From the definition of $E_{c}$, Eq. (6), and the proof of Lemma 1, we have that there exists a $k>N$ as well as $m$ and $\mathcal{L}$ such that

$$
\left|E_{c}(\rho)-\frac{m}{k}\right| \leq \Delta
$$

and $D\left(\rho^{\otimes k}, \rho_{k}\right)<\epsilon$, where $\rho_{k}=\mathcal{L}\left(\left|\Psi_{-}\right\rangle\left\langle\left.\Psi_{-}\right|^{\otimes m}\right)\right.$.

Next, we can bound

$$
\left|\lim A_{n}-\frac{E_{f}\left(\rho_{k}\right)}{k}\right| \leq\left|\lim A_{n}-A_{k}\right|+\left|A_{k}-\frac{E_{f}\left(\rho_{k}\right)}{k}\right|<2 \Delta,
$$

by using Eqs. (15) and (16).

This gives $E_{f}\left(\rho_{k}\right)>k\left(\lim A_{n}-2 \Delta\right)$. On the other hand, by Eq. (17), we have

$$
E_{f}\left(\left|\Psi_{-}\right\rangle\left\langle\left.\Psi_{-}\right|^{\otimes m}\right)=m<k\left(E_{c}(\rho)+\Delta\right),\right.
$$

which finally yields

$$
E_{f}\left(\rho_{k}\right)-E_{f}\left(\left|\Psi_{-}\right\rangle\left\langle\left.\Psi_{-}\right|^{\otimes m}\right)>k\left(\lim A_{n}-E_{c}(\rho)-3 \Delta\right)=k \Delta,\right.
$$

a contradiction since the entanglement of formation cannot increase under LOCC operations. 


\section{ALTERNATIVE DEFINITIONS OF THE ASYMPTOTIC COST}

While $E_{c}(\rho)$ is perhaps the most natural function to associate with the asymptotic cost of preparing a bipartite state, other definitions would have been consistent with our discussion in the introduction. An example of a different but perhaps useful definition of the asymptotic entanglement cost is the following:

$$
E_{\text {alt }}(\rho)=\inf \left\{E|\forall \epsilon>0, \delta>0, \exists N|\left(\forall n>N \exists m, \mathcal{L},\left|E-\frac{m}{n}\right| \leq \delta \text { and } D\left(\mathcal{L}\left(\left|\Psi_{-}\right\rangle\left\langle\left.\Psi_{-}\right|^{\otimes m}\right), \rho^{\otimes n}\right) \leq \epsilon\right)\right\} .\right.
$$

The difference between $E_{c}$ and $E_{a l t}$ is that for fixed fidelity and cost the former only requires the existence of a single $n$ such that $\rho^{\otimes n}$ can be approximated efficiently while the second requires that there exist a threshold $N$ such that for all $n>N$ the state $\rho^{\otimes n}$ can be approximated efficiently. One consequence of this difference is that it is immediately clear that $E_{\text {alt }}$ is additive in this sense of Lemma 1, while some work was required to prove that $E_{c}$ was. Indeed, a priori, one might not expect the two definitions to agree. Suppose, for example, that for fixed $\epsilon$ there exists a protocol to make an approximation $\rho_{n}$ of $\rho^{\otimes n}$ from $m$ singlets, with the approximation satisfying $D\left(\rho_{n}, \rho^{\otimes n}\right)=\epsilon$. In order to produce an approximation to $\rho^{\otimes n k}$ from $k m$ singlets, one might think that applying this protocol $k$ times would be a good strategy. One finds, however, that for $\epsilon>0$,

$$
\lim _{k \rightarrow \infty} D\left(\rho^{\otimes n k}, \rho_{n}^{\otimes k}\right)=\lim _{k \rightarrow \infty} 2 \sqrt{1-\left(1-\epsilon^{2}\right)^{k}}=2 .
$$

The protocol, therefore, generally fails to produce a good approximation for large $k$. This example suggests that the existence of a threshold $N$ beyond which approximations to some given fidelity are always possible is a very difficult condition to satisfy. Nonetheless, by applying the results of the previous section, it is actually easy to see that the definitions $E_{c}$ and $E_{a l t}$ are equivalent, so that the extra condition can be met without increasing the asymptotic unit cost. First, notice that the argument of Lemma 2 actually also works for $E_{\text {alt }}$ so that $E_{\text {alt }}(\rho) \leq E_{f}^{\infty}(\rho)$. Next, since the definition of $E_{\text {alt }}$ is more stringent than that of $E_{c}$, we have that $E_{c}(\rho) \leq E_{\text {alt }}(\rho)$. Combining these inequalities with the result of Lemma 3, we get

$$
E_{f}^{\infty}(\rho) \leq E_{c}(\rho) \leq E_{a l t}(\rho) \leq E_{f}^{\infty}(\rho)
$$

so that these two definitions of the entanglement cost always agree.

A final pair of alternative definitions for the asymptotic entanglement cost would use the trace distance

$$
d(\rho, \sigma)=\frac{1}{2} \operatorname{Tr}|\rho-\sigma|,
$$

in place of the Bures distance. Lemma 1 is easily seen to hold for $d$ since its proof uses only axiomatic properties of all metrics and stability with respect to tensor products but no other properties specific to $D$. The status of Lemmas 2 and 3 is slightly more involved. The trace distance and Bures distance are related by the following inequalities [22]

$$
\frac{1}{4} D(\rho, \sigma)^{2}=1-F(\rho, \sigma) \leq d(\rho, \sigma) \leq \sqrt{1-F(\rho, \sigma)^{2}}=\sqrt{\frac{D^{2}}{2}-\frac{D^{4}}{16}},
$$

which show that $d$ and $D$ are equivalent metrics and bounds them in terms of each other by functions that are independent of the dimension of the underlying Hilbert space, $\mathcal{H}$. In other words, $d$ and $D$ are equivalent metrics even in the asymptotic regime. To be concrete, applying the right-hand inequality to Eq. (13), for example, is sufficient to show that Lemma 2 also holds for the trace distance. In order to prove Lemma 3, we used the continuity relation, Eq. (3), but effectively only required the weaker inequality

$$
|E(\rho)-E(\sigma)| \leq B D(\rho, \sigma) \log \operatorname{dim} \mathcal{H}+C,
$$

where $B$ and $C$ are constants and $\mathcal{H}$ is the supporting Hilbert space. Applying Eq. (25), however, gives an inequality of the form

$$
|E(\rho)-E(\sigma)| \leq B \sqrt{d(\rho, \sigma)} \log \operatorname{dim} \mathcal{H}+C,
$$

which again is sufficient to carry through the rest of the proof. Therefore, all our conclusions hold even if the entanglement cost is defined using the trace distance. Indeed, they should hold for any metric equivalent to the Bures metric in which the equivalence is given by a function that is independent of the dimension of $\mathcal{H}$. 


\section{CONCLUSIONS}

We have given two rigorous definitions of the asymptotic cost of preparing a bipartite mixed state $\rho$ and shown them both to be equal to the regularized entanglement of formation, $E_{f}^{\infty}(\rho)$, resolving an important conjecture in the theory of quantum entanglement. Furthermore, we have shown that this asymptotic cost is fairly insensitive to the choice of metric on density operators. In particular, the Bures distance and trace distance result in identical asymptotic costs.

An important problem left open by this work is the question of actually evaluating $E_{f}^{\infty}(\rho)$. Even the non-regularized function $E_{f}(\rho)$ is notoriously difficult to calculate; its value is only known for some very special cases 国,23]. If it turns out that $E_{f}$ is not additive for tensor products, then, in spite of the results of this paper, determining the asymptotic cost of preparing a state remains quite a formidable problem.

\section{ACKNOWLEDGMENTS}

We would like to thank Paweł Horodecki, Michael Nielsen and John Smolin for helpful discussions. PMH is grateful to the Rhodes Trust and the EU project QAIP, contract No. IST-1999-11234, for support. MH acknowledges support of Polish Committee for Scientific Research, contract No. 2 P03B 10316 and EU project EQUIP, contract No. IST-1999-11053. BMT acknowledges support of the ARO under contract number DAAG-55-98-C-0041.

[1] C.H. Bennett, D.P. DiVincenzo, J.A. Smolin and W.K. Wootters, Mixed state entanglement and quantum error correction, Phys. Rev. A 54, 3824-3851 (1996), quant-ph/9604024.

[2] C. H. Bennett, G. Brassard, S. Popescu, B. Schumacher, J. Smolin and W. K. Wootters, Purification of noisy entangelment and faithful teleportation via noisy channels, Phys. Rev. Lett. 76, 722 (1996), quant-ph/9511027.

[3] C.H. Bennett, H.J. Bernstein, S. Popescu and B. Schumacher, Concentrating partial rntanglement by local operations, Phys. Rev. A 53, 2046 (1996), quant-ph/9511030.

[4] W.K. Wootters, Entanglement of formation of an arbitrary state of two qubits, Phys. Rev. Lett. 80, 2245 (1998), quant$\mathrm{ph} / 9709029$.

[5] P. Horodecki, M. Horodecki and R. Horodecki, Entanglement and thermodynamical analogies, Acta Phys. Slovaca 48, 141 (1998).

[6] E. M. Rains, Rigorous treatment of distillable entanglement, Phys. Rev. A 60, 173 (1999), quant-ph/9809078.

[7] B. Schumacher, Quantum coding, Phys. Rev. A 51, 2738 (1995).

[8] M.A. Nielsen, Continuity bounds for entanglement, Phys. Rev. A 61, 064301 (2000), quant-ph/9908086.

[9] M. Horodecki, P. Horodecki and R. Horodecki, Limits for entanglement measures, Phys. Rev. Lett. 84, 2014 (2000).

[10] M. Horodecki, P. Horodecki and R. Horodecki, Asymptotic entanglement manipulations can exhibit genuine irreversibility Phys. Rev. Lett. 84, 4260 (2000); Unified approach to quantum capacities: towards quantum noisy coding theorem, ibid, $\mathbf{8 4}, 4260(2000)$.

[11] A. Uhlmann, The 'transition probability' in the state space of a *-algebra, Rep. Math. Phys. 9, 273 (1976).

[12] R. Jozsa, Fidelity for mixed quantum states, J. Mod. Opt. 41, 2315 (1994).

[13] M. Donald and M. Horodecki, Continuity of relative entropy of entanglement, Phys. Lett. A 264, 257 (1999).

[14] V. Vedral, M. B. Plenio, M. A. Rippin and P. L. Knight, Quantifying entanglement, Phys. Rev. Lett. 78, 2275 (1997), quant-ph/9702027.

[15] C. H. Bennett, S. Popescu, D. Rohrlich, J. A. Smolin and A. V. Thapliyal, Exact and asymptotic measures of multipartite pure state entanglement, quant-ph/9908073.

[16] M. Keyl, R. F. Werner, The rate of the optimal purification procedures, quant-ph/9910124.

[17] See e.g. H. Barnum, M. Nielsen and B. Schumacher, Information transmission through a noisy quantum channel, Phys. Rev. A 57, 4153 (1998), quant-ph/9702049.

[18] Note that we are using an approximation to $\rho^{\otimes n}$ which is based on the optimal decomposition for the entanglement of formation, and not on the eigendecomposition of $\rho$ which is used in Schumacher compression [⿰]]. Both approximations converge to $\rho^{\otimes n}$ in the limit of large $n$.

[19] T.M. Cover and J.A. Thomas, Elements of Information Theory, (John Wiley \& Sons, 1991).

[20] D. Jonathan and M. Plenio, Minimal conditions for local pure-state entanglement manipulation, Phys. Rev. Lett. 83, 1455 (1999).

[21] P. Hayden, B.M. Terhal and A. Uhlmann, in preparation. 
[22] C. A. Fuchs and J. van de Graaf, Cryptographic distinguishability measures for quantum mechanical states, IEEE T. Inform. Theory 45: (4) 1216 (1999), quant-ph/9712042.

[23] B. M. Terhal and K. G. H. Vollbrecht, The entanglement of formation for isotropic states, quant-ph/0005062. 